\title{
Examples of trapped modes in the presence of freely-floating structures
}

\author{
By R. PORTER AND D. V. EVANS
}

School of Mathematics, University of Bristol, Bristol, BS8 1TW, UK

(Received 13 August 2007)

A freely floating motion trapping structure can be defined as one or more rigid bodies floating on the surface of a fluid which extends to infinity in at least one direction, whose free motion under its natural hydrostatic restoring force is coupled to that of the surrounding fluid in such a way that no waves are radiated to infinity. The resulting local time-harmonic oscillation of the structure and the surrounding fluid is called a motion trapped mode. Such a structure would, if displaced slightly from its equilibrium position and let go, ultimately oscillate indefinitely at the trapped mode frequency. Previous examples of motion trapping structures have been devised using an inverse approach in which the shape of pairs of such structures are determined implicitly by sketching certain streamlines. In this paper an alternative direct approach to the construction of motion trapping structures in the form of a pair of identical floating cylinders of rectangular crosssection in two dimensions is presented. It is also shown that a thick-walled axisymmetric heaving circular cylinder can act as a motion trapping structure.

\section{Introduction}

The classical linear theory of the interaction of waves with fixed or floating bodies has presented challenging theoretical problems for over 150 years. One of the most fundamental of these is the question of the uniqueness of solutions to the governing equations. While such questions can be expressed succinctly in the terminology of spectral theory for unbounded operators, a more physical interpretation of a non-uniqueness is the existence of a local bounded oscillation at a well-defined frequency in the vicinity of a fixed rigid body or bodies on or under a free surface extending to infinity in at least one direction. Although such oscillations or trapped modes were known to exist in a wide 
class of three-dimensional problems, it was generally believed that they did not exist in two dimensions and this was confirmed by a number of partial results usually relating to single fixed bodies. However McIver (1996) was able to show, using an inverse method, that certain pairs of fixed surface-piercing two-dimensional cylinders were able to support trapped modes of a particular frequency. Since then numerous examples of fixed trapping structures have been discovered in both two and three dimensions. For a fairly recent survey, see Evans \& Kuznetsov (1997).

A key feature of these trapped modes is that they occur in the presence of fixed bodies. However, recently interest has shifted to the question as to whether motion trapped modes arise where the body is free to move under its natural or externally applied restoring force at the same frequency as the localized trapped mode. In the absence of viscous damping, such a motion trapping structure would, given an initial displacement or velocity from its equilibrium state, eventually settle down to a simple harmonic motion at the trapped mode frequency. Recently the authors provided the first example of a submerged motion trapping structure in the form of a single two-dimensional buoyant circular cylinder tethered to the bottom in fluid of finite depth (Evans \& Porter 2007). The construction made use of the fact, previously overlooked, that the waves radiated to infinity by the forced motion of such a cylinder could vanish at certain depths of submergence and wave frequency, a necessary condition for a motion trapping structure. At that frequency, it also proved possible to satisfy the second condition for trapping which is that there should be a balance between the inertia forces on the cylinder, including that due to the surrounding fluid, and any linearised restoring forces, in this case the component of the tension in the tether acting on the cylinder.

A more natural motion trapping structure is one which is freely floating and where the only restoring force is hydrostatic and most attention has gone into finding examples of this type. Notable in this regard is the work of McIver \& McIver (2006) who first derived the conditions described above for a motion trapping structure. They showed that for freely floating structures under hydrostatic restoring forces only, the second condition for trapping could be replaced by the requirement that the dipole moment for the potential describing the motion should vanish at infinity. They used an inverse method similar to that used by McIver (1996) described above, to construct potentials which were both wave free and satisfied the zero dipole moment condition. By sketching the corresponding streamlines they were able to construct 'mirror image' pairs of identical freely floating 
bodies as trapping structures. Subsequently (McIver \& McIver 2007), they were able to extend the idea to construct an axisymmetric freely floating torus of a specific shape which acted as a motion trapping structure. In this paper we shall also seek freely floating trapping structures under hydrostatic forces but we shall take a different more direct approach which provides a constructive technique to finding pairs of floating bodies of simple shapes which can support motion trapping.

The examples of McIver \& McIver $(2006,2007)$ and those we shall construct here share the property that there is an internal portion of the free surface, bounded laterally by a surface piercing structure. In the naval hydrodynamics community such configurations are often referred to as "moonpools" and interest often centres on the location and nature of near resonances. In the current context of freely-floating structures the most relevant work is that of Wang \& Wahab (1971), who investigated the free heaving of a pair of cylinders floating in the free surface, and Yeung \& Seah (2007), which is explained in more detail in the next paragraph.

The main focus of this paper is on the two-dimensional interaction of waves with floating cylinders of rectangular cross-section, although we extend this to consider threedimensional wave interaction with a floating axisymmetric shell with a rectangular wall cross-section. Various hydrodynamic problems associated with each geometrical configuration need to be solved. In each case, this is achieved using a domain decomposition approach, in which the solution in each fundamental rectangular domain is represented by the appropriate eigenfunction expansion. This is a standard and practical approach to take. Indeed it has been implemented most recently by Yeung \& Seah (2007) whose geometrical configuration is identical to one of those we consider here. However, we take a different approach to formulating each solution, choosing to derive integral equations in the matching process, rather than matching mode-by-mode as in Yeung \& Seah (2007). The integral equations are then solved using a Galerkin approximation which involves taking explicit account of the singularity in the velocity field at any sharp corners in the rectangular geometry which occur at the extremities of the matching intervals. A similar approach can be found in Porter (1995) and Evans \& Fernyhough (1995), for example. Although arguably more complicated to formulate, the integral equation approach has some significant advantages over the mode-matching approach taken by Yeung \& Seah (2007). Principally, we can guarantee a high degree of accuracy in the computation of the hydrodynamic coefficients (at least five significant figures) by inverting relatively small 
systems of equations (i.e. less than 10 by 10). In contrast, mode-matching is less accurate and requires the inversion of much larger systems. The degree of accuracy is critical in providing conclusive numerical evidence of the existence of motion trapped modes, as the balance between various hydrodynamic coefficients needed for this is rather subtle (see the discussion in section 5).

Since the details of the formulation of the three hydrodynamic problems is rather complicated, and the methods are well-established in the literature, we have decided to list in Appendices A, B and C the essential ingredients that are required to produce our numerical results.

The plan of the paper is as follows. In the next section, we provide an argument for the generation of motion trapping structures, based on the existence of isolated bodies which are capable of reflecting all incident wave energy. In section 3, we determine criteria for total reflection of isolated structures moving in free response to the surrounding fluid, based on standard hydrodynamic coefficients, namely added mass, damping, reflection and transmission coefficients. In section 4 , the theory is applied to floating rectangular cylinders and in section 5, pairs of cylinders are considered and the exact configurations are compared with those predicted via the theory of section 2. Three-dimensional axisymmetric structures are also considered. Finally, some conclusions are drawn in section 6.

\section{Arguments for the construction of motion trapping structures}

The two conditions derived by McIver \& McIver (2006) which need to be satisfied simultaneously at a given frequency for a motion trapping structure, in either two or three dimensions, are

$$
(M+a(\omega)) \omega^{2}-\lambda=0
$$

and

$$
b(\omega)=0 .
$$

Here $a(\omega), b(\omega)$ are, respectively, the added mass and radiation damping coefficients for the structure, $M$ is the mass of the structure, and $\lambda=\rho g W$ where $W$ is the waterplane area of the structure. We shall be concerned with the construction of trapping structures in the form of a pair of identical heaving cylinders in two dimensions, and their counterpart in three dimensions, a heaving axisymmetric thick cylinder. Much of the argument 
will be based on the two-dimensional catamaran structure which is where we begin. Consider the requirement that the radiation damping should vanish for the heaving cylinder pair. If that were possible at a particular frequency the fluid motion between the cylinders would be a standing wave at that frequency with a local evanescent field external to the cylinders. The same argument would apply if the two cylinders were widely-spaced in which case it is possible to apply a wide-spacing argument. Thus consider an incident wave from infinity which excites a single freely-floating heaving cylinder giving rise to reflected and transmitted waves. If now for a particular frequency, the transmission coefficient $T_{1}$ vanishes, the incident wave will be totally reflected so that the reflection coefficient $R_{1}$ satisfies $\left|R_{1}\right|=1$. We now imagine an identical freely-floating cylinder a large distance $2 b$ upstream of the first, moving as its mirror image in the line midway between them. The reflected wave will in turn be totally reflected and it is easily shown that a symmetric standing wave or motion trapped mode can be set up if the spacing satisfies

$$
k b=-\frac{1}{2} \arg \left\{R_{1}\right\}+n \pi
$$

where $k$ is the wavenumber (equal to $K \equiv \omega^{2} / g$ in deep water and defined by $K=$ $k \tanh k h$ in water of depth $h$ ), and $n$ is an integer. It is clear that taken together the two freely heaving cylinders constitute a motion trapping structure. Thus the widespacing argument, which neglects the influence of the local field near one cylinder on the other, has shifted the problem to finding single cylinders which, when freely floating in response to an incident wave from infinity, are capable of totally reflecting that wave at a particular frequency. Once such a cylinder has been found, then (2.3) provides an approximate formula for the spacing required between two such cylinders to constitute a trapping structure, in terms of the phase of the reflection coefficient at that frequency. In addition the formula (2.3) provides a starting point for determining the location of motion trapped modes when the cylinders move closer together and local effects can no longer be ignored. We need to find conditions under which a single cylinder, free to move in a single mode of motion, can totally reflect the incident wave which is exciting it. These conditions were derived by Evans \& Linton (1989) who were investigating the efficacy of a submerged buoyant tethered circular cylinder swaying freely in response to an incident wave field as an active wave reflector. The analysis is repeated in the next section for an arbitrary cylinder, symmetric about a vertical line, which is free to heave in the presence of an incident wave field. 


\section{Scattering by a cylinder in free response to an incident wave}

Two-dimensional Cartesian coordinates $(x, z)$ are taken with the origin in the mean free surface of the fluid and the $z$-axis pointing downwards with $z=h$ aligned with the bottom of the fluid, which has density $\rho$. We consider a single cylinder of mass per unit length $M$ floating in the surface, free to move in heave response to incident plane waves of unit amplitude from $x=\infty$ of angular frequency $\omega$. Then, assuming a time-dependence of $\mathrm{e}^{-i \omega t}$, the time-independent velocity potential, $\phi(x, z)$ satisfies the usual linearised equations of motion,

$$
\nabla^{2} \phi=0, \quad \text { in the fluid, } \quad \frac{\partial \phi}{\partial z}=K \phi, \quad \text { on } z=0
$$

where $K=\omega^{2} / g$ ( $g$ being acceleration due to gravity) with the cylinder boundary condition

$$
\hat{\mathbf{n}} \cdot \nabla \phi=\hat{\mathbf{n}} \cdot \mathbf{U}
$$

where $\hat{\mathbf{n}}$ is the outward unit normal and $\mathbf{U}=U \hat{\mathbf{z}}$ for heave oscillations. Far from the cylinder, the potential is described by

$$
\phi(x, z) \sim\left\{\begin{array}{l}
(g / \omega)\left(\mathrm{e}^{-\mathrm{i} k x}+R_{1} \mathrm{e}^{\mathrm{i} k x}\right) \psi_{0}(z), \quad x \rightarrow+\infty \\
(g / \omega) T_{1} \mathrm{e}^{-\mathrm{i} k x} \psi_{0}(z) \quad x \rightarrow-\infty
\end{array}\right.
$$

where $\psi_{0}(z)$ represents the vertical variation of the velocity potential associated with propagating waves and $k$ is the positive real root of the dispersion relation $K=k \tanh k h$, which reduces to $K=k$ in infinite depth. It is our aim, in this section to find the reflection and transmission coefficients, $R_{1}$ and $T_{1}$.

Because of the linearity of the governing equations, we write

$$
\phi=U \phi_{r}+\phi_{s}
$$

where $\phi_{r}$ is the radiation potential for unit forced heave velocity in the absence of the incident wave, and $\phi_{s}$ is the scattered potential due to a unit amplitude wave incident from $x=+\infty$ on the cylinder when it is assumed to be held fixed. The constant, $U$, in (3.4) is the unknown velocity in heave, to be determined from the equation of motion of the cylinder.

Thus we have

$$
\phi_{r} \sim A_{h} \mathrm{e}^{\mathrm{i} k|x|} \psi_{0}(z), \quad|x| \rightarrow \infty
$$

where $A_{h}$ is related to the amplitude of outgoing waves from the cylinder where $\hat{\mathbf{n}} \cdot \nabla \phi_{r}=$ 
$\hat{\mathbf{n}} . \hat{\mathbf{z}}$ applies to the wetted surface of the cylinder. Also, $\hat{\mathbf{n}} . \nabla \phi_{s}=0$ and

$$
\phi_{s} \sim\left\{\begin{array}{l}
(g / \omega)\left(\mathrm{e}^{-\mathrm{i} k x}+R \mathrm{e}^{\mathrm{i} k x}\right) \psi_{0}(z) \quad x \rightarrow \infty \\
(g / \omega) T \mathrm{e}^{-\mathrm{i} k x} \psi_{0}(z) \quad x \rightarrow-\infty
\end{array}\right.
$$

where $R$ and $T$ are the reflection and transmission coefficients for the fixed cylinder.

The coefficients $A_{h}, R$ and $T$ are determined from the boundary-value problems specified for $\phi_{r}$ and $\phi_{s}$ and depend upon frequency.

It follows from the above definitions that

$$
\left.\begin{array}{l}
R_{1}=R+(\omega U / g) A_{h} \\
T_{1}=T+(\omega U / g) A_{h}
\end{array}\right\}
$$

in which the cylinder velocity, $U$, still needs to be determined.

We use the equation of motion for a cylinder of mass per unit length $M$,

$$
F_{e x t}+F_{r}+F_{s}=-\mathrm{i} \omega M U
$$

where the time-independent forces acting on the cylinder are $F_{s}$, the vertical wave exciting force on the fixed cylinder, $F_{r}$, the vertical wave radiation force on the cylinder due to its own motion, and $F_{\text {ext }}$, the external force which is simply the vertical hydrostatic force.

Thus, we write $F_{\text {ext }}=-\mathrm{i} \lambda \omega^{-1} U$ with $\lambda=\rho g W$ where $W$ is the waterplane area for a cylinder intersecting the free surface. The wave radiation force is decomposed into components in phase with the acceleration and velocity of the body, through

$$
F_{r}=-(b(\omega)-\mathrm{i} \omega a(\omega)) U
$$

where $a(\omega)$ and $b(\omega)$ are the added mass and radiation damping coefficients, respectively, of the cylinder in forced heave motion.

The heave exciting force is not calculated directly. Instead, use is made of a number of useful reciprocal relations which exist for radiation and scattering problems involving the potentials $\phi_{r}$ and $\phi_{s}$. For example the Haskind relation connects the vertical exciting force on the fixed cylinder in incident waves to the amplitude at $x=\infty$ due to unit heave velocity of the cylinder. Thus

$$
F_{s}=\rho g A_{h} d(k h)
$$

where $d(k h)$ is a depth-dependent term whose explicit definition is unimportant. Next we have the relation between the radiation damping coefficient and the far field amplitude,

$$
b(\omega)=\rho \omega\left|A_{h}\right|^{2} d(k h)
$$


and finally the Newman relation states

$$
R+T=-A_{h} / \overline{A_{h}}
$$

connecting the scattering coefficients and the phase of the far-field radiated heave amplitude.

Use of the definition (3.9) and that for $F_{\text {ext }}$ in (3.8) now shows that

$$
b(\omega)(1-\mathrm{i} C) U=F_{s}
$$

where

$$
C=\left((M+a(\omega)) \omega^{2}-\lambda\right) /(b(\omega) \omega) .
$$

Elimination of $F_{s} / b(\omega)$ in (3.13) via the reciprocal relations (3.10)-(3.11), followed by use of (3.12), determines the heave velocity as

$$
U=-\frac{g}{\omega A_{h}} \frac{(R+T)}{(1-\mathrm{i} C)}
$$

and finally use in (3.7) gives

$$
\left.\begin{array}{l}
R_{1}=(C R-\mathrm{i} T) /(C+\mathrm{i}) \\
T_{1}=(C T-\mathrm{i} R) /(C+\mathrm{i})
\end{array}\right\} .
$$

It follows that

$$
\left|R_{1}\right|^{2}+\left|T_{1}\right|^{2}=|R|^{2}+|T|^{2}=1
$$

since $|R \pm T|=1$ for vertically symmetric bodies and $R / T=\mathrm{i} \chi$ where $\chi$ is real. Thus $T_{1}=T(C+\chi) /(C+\mathrm{i})$ and clearly $T_{1}=0$ provided

$$
C=-\chi, \quad \text { or } \quad(M+a(\omega)) \omega^{2}-\lambda=-b(\omega) \omega \chi .
$$

It is noteworthy that the above analysis is exact and that the far field radiated by a freely-floating cylinder responding to a given incident wave field, can be determined explicitly in terms of the scattered field from a fixed cylinder in that incident field, and the radiated field due to the forced motion of the cylinder. A similar analysis can be derived for a cylinder moving freely in either sway or roll, when the condition for $T_{1}$ to vanish is obtained by replacing $-\chi$ by $\chi$ in (3.18). In this case there is no vertical hydrostatic restoring force so that $\lambda$ would correspond to any external restoring force. This was the case considered by Evans \& Linton (1989). 

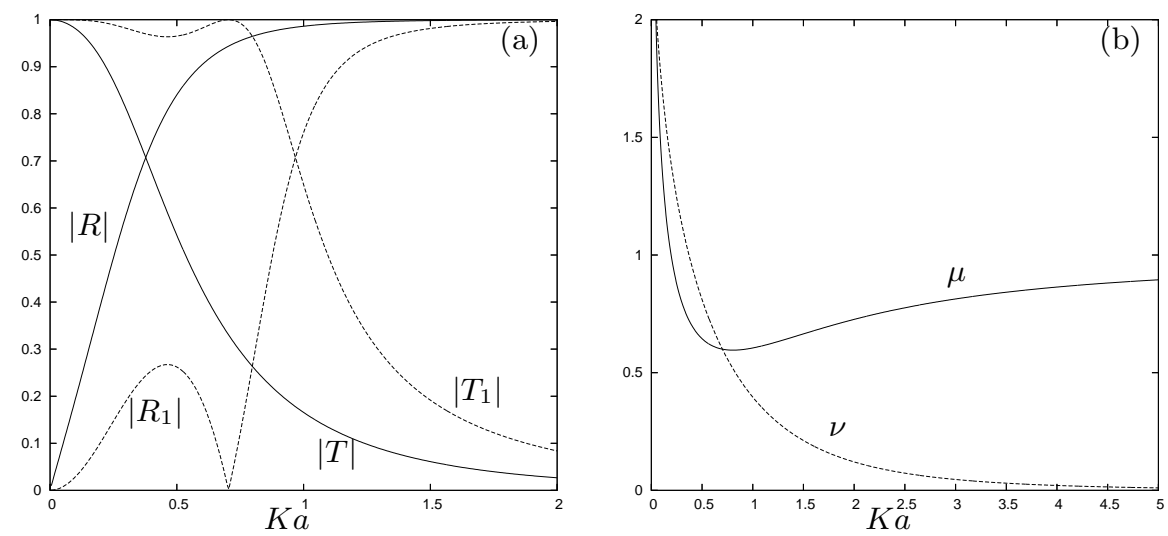

FiguRE 1. Variation of (a) $|R|,|T|$ for a fixed cylinder and $\left|R_{1}\right|,\left|T_{1}\right|$ for a freely-heaving cylinder and (b) $\mu, \nu$, the non-dimensional heave added-mass and radiation damping against $K a=\omega^{2} a / g$. The cylinder is semi-immersed in the free surface. There are no zeroes of transmission.

\section{Freely floating cylinders of circular and rectangular cross-sections}

In the case of the freely floating cylinder constrained to move in heave, the required condition for $T_{1}$ to vanish is equation (3.18) where the opposing hydrostatic force gives $\lambda=2 a \rho g$ where $2 a$ is the width of the cylinder at the water surface. There would appear to be no reason why (3.18) should be satisfied in any particular case. To begin with a half-immersed floating semicircular cylinder was considered in infinite depth fluid using multipoles (see Martin \& Dixon (1984) for details) and computations of $R, T$, and hence $\chi$, and $a(\omega), b(\omega)$ were made, from which $C$ could be computed and the condition (3.18) checked. The curves of reflection and transmission coefficients against $K a$ for a fixed cylinder, a cylinder allowed to respond freely in heave are shown in figure 1(a), whilst the non-dimensional heave added-mass, $\mu=a(\omega) / M$ and radiation damping $\nu=b(\omega) / M \omega$ (where $M=\frac{1}{2} \rho \pi a^{2}$ is the mass of the cylinder) are shown alongside in figure 1(b). Thus, it can be seen that $T_{1}$ fails to vanish at any value of $K a$. However, it has been demonstrated by the authors that an extension of the analysis in the preceding section to allow free motion in both heave and sway, applied to the semi-immersed circular cylinder does give rise to zeroes of transmission. This result will form the subject of a separate paper.

The hydrodynamic coefficients for the half-immersed cylinder in infinite depth depend upon a single parameter, $\mathrm{Ka}$. It was therefore decided to consider the freely floating vertical rectangular cylinder which possesses an extra degree of freedom in the draft of the cylinder which could be varied and hence make it more likely that equation (3.18) could be satisfied and a zero of $\left|T_{1}\right|$ found. There are strong reasons to expect that zeroes 
of transmission occur in this case. In fact such total reflection has been shown to exist, albeit indirectly, in relation to a different problem, when the rectangle is narrow. Thus Evans \& Morris (1972) proved that two vertical partially-immersed fixed barriers could totally reflect an incident wave of a certain frequency for certain values of the geometric parameters, and they provided the frequency bands within which this would occur. The more common phenomenon of total transmission was also shown, but the vanishing of the transmission coefficient was new. Subsequently Newman (1974) confirmed these results using a matched asymptotic expansion method valid for closely-spaced barriers in which he matched the far field potential with a local potential valid near the barriers and up to the interior free surface between them. Physically this local solution described a uniform slug flow of the fluid between the barriers acting as a rigid body. Thus the solution could also be interpreted as the total reflection of an incident wave by a narrow rectangular cylinder constrained to move in heave.

A description of the full linear solution for scattering of an incident wave from - and waves radiated by - a vertical rectangular cylinder in finite depth is given in Appendix A. All the hydrodynamic coefficients necessary for determining $T_{1}$ for the freely floating cylinder in addition to the real expression $C+\chi$, we computed for varying geometric parameters and the dimensionless wavenumber, $k d$. For a fixed set of geometrical parameters, it is a simple matter to determine when $T_{1}=0$, as it corresponds to the point at which the function $C+\chi$, crosses the $k d$ axis. The non-dimensional form for $C$ is

$$
C=\left[(1+\mu)-(K d)^{-1}\right] / \nu
$$

where $\mu=a(\omega) / M, \nu=b(\omega) /(\omega M)$ where $M=2 \rho a d$ is the mass of the rectangular cylinder, coinciding with the definitions in Appendix A.

A sample set of results is given in figures 2(a)-(c) where curves showing the variation of $|T|$ for a fixed rectangular cylinder, $\mu$ and $\nu$ for a heaving rectangular cylinder are plotted against $k d$ for a range of values of cylinder aspect ratio, $a / d$. The draft to water depth ratio is fixed at $d / h=0.2$. These results are combined according to equation (3.16) to give the curves for $\left|T_{1}\right|$ shown in figure 2(d). Although the curves in figures 2(a)-(c) display no remarkable features, curves of $\left|T_{1}\right|$ possess a much more interesting behaviour. In particular, it is just about possible to make out zeroes of $T_{1}$ when $a / d$ takes both small $(0.2,0.5)$ and large (4) values. The difficulty in discerning zeroes of $T_{1}$ when $a / d$ equals 1 and 2 is made evident in the comprehensive plot in figure 3(a). Here, we trace zeroes of $T_{1}$ in $(a / d, k d)$ parameter space, with each curve now corresponding to a different choice 

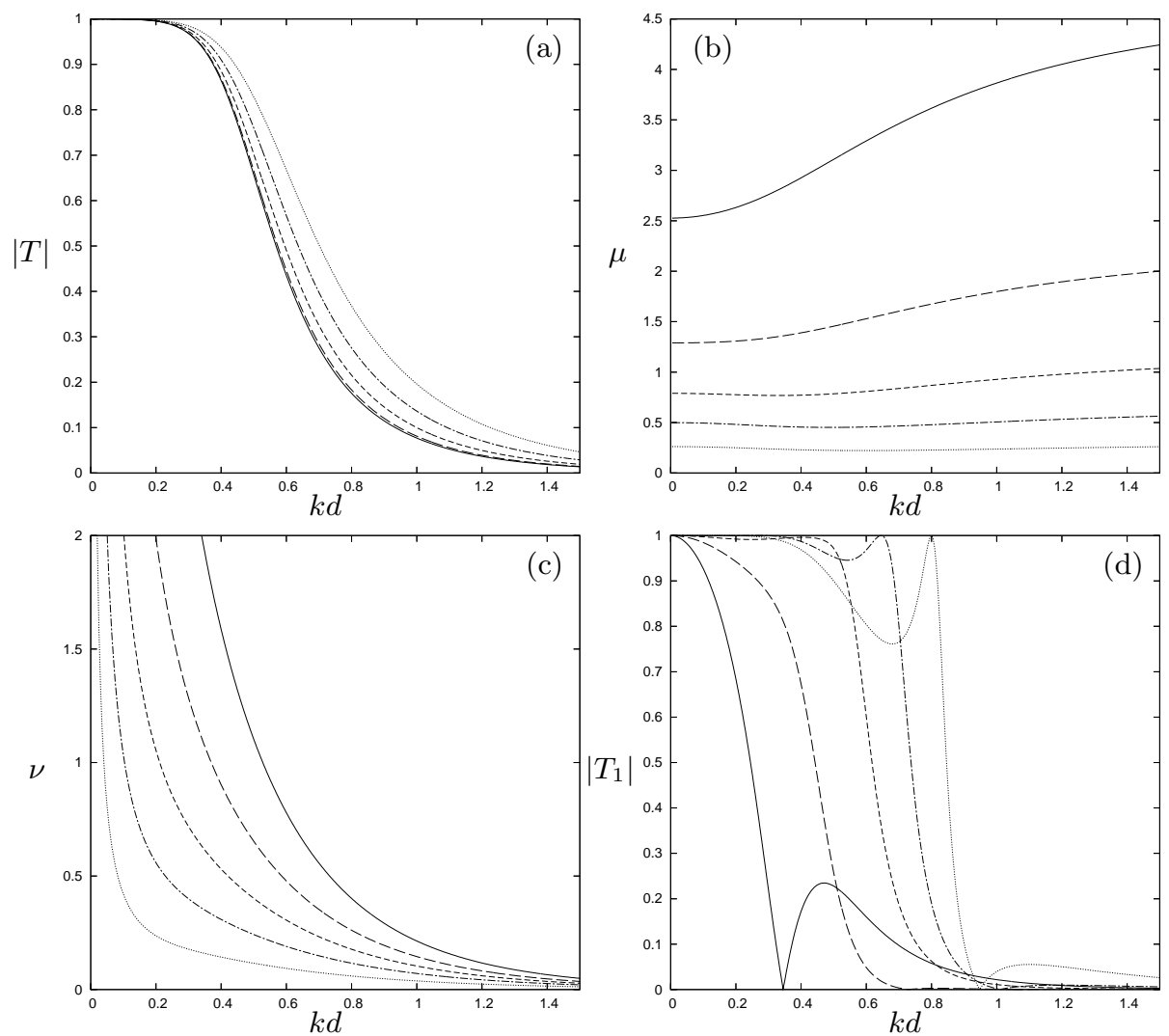

FIgURE 2. Variation with non-dimensional wavenumber, $k d$, of: (a) $|T|$ the transmission coefficient for a fixed cylinder; (b) added mass, $\mu$, and (c) radiation damping, $\nu$, for a heaving cylinder; (d) $\left|T_{1}\right|$ for a freely-heaving cylinder. Draft to depth ratio, $d / h=0.2$. Curves show $a / d$ of 4 (solid), 2 (long dashed), 1 (short dashed), 0.5 (chained), 0.2 (dotted).

of depth ratio $d / h$, ranging in values from 0.1 to 0.3 . Thus, for $d / h=0.2$, it can be seen that zeroes of $T_{1}$ exist only for $a / d<0.824$ and $a / d>1.917$. What is fascinating about the results shown in figure 3(a) is the sensitive dependence on the depth of the fluid, with a value of $d / h \approx 0.226$ dividing the cylinder geometries into two regimes: those which possess zeroes of transmission for all lengths of cylinder and those which do not. Note also that where zeroes of $T_{1}$ exist they always occur at two values of $k d$ for each value of $a / d>0$. This follows since it can be shown that $C+\chi<0$ (where it transpires that $\chi=-|R| /|T|)$ for both large and small values of $k d$, so that it must vanish an even number of times which just happens to be twice.

With reference to the earlier discussion in which the search for zeroes of $T_{1}$ for thin rectangular cylinders was motivated, attention is drawn to the curve corresponding to $a / d=0.2$ in figure $2(\mathrm{~d})$, which undergoes a sharp deviation from $\left|T_{1}\right|=1$ to $\left|T_{1}\right|=0$, 

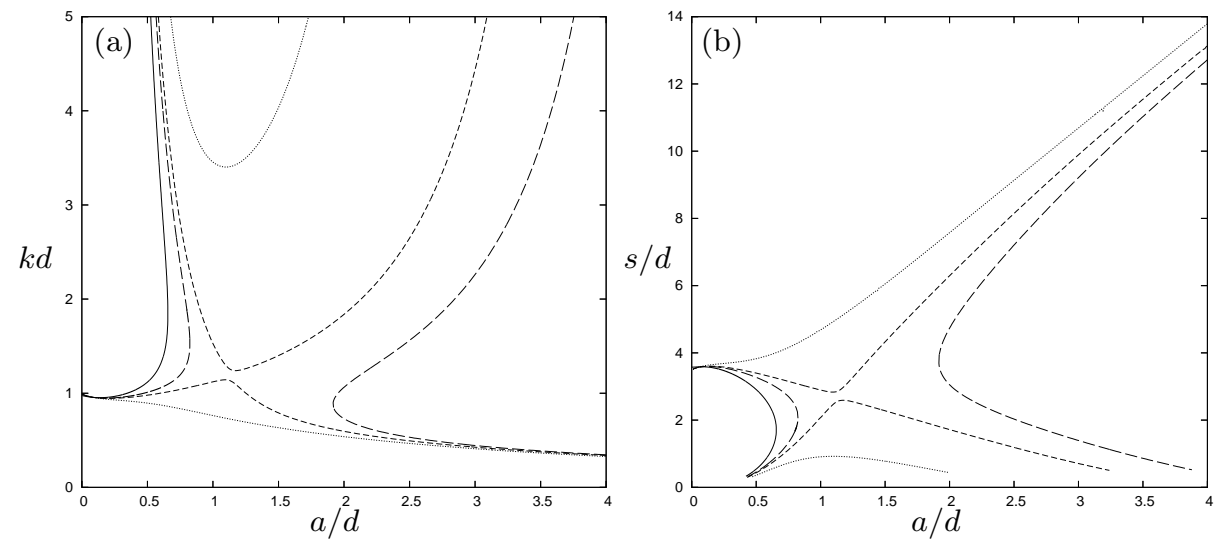

Figure 3. In (a), the location of zeroes of $T_{1}$ in $(k d, a / d)$ parameter space, with depth ratios $d / h=0.1$ (solid), 0.2 (long dash), 0.226 (short dash) and 0.3 (dotted). In (b), the predicted half-spacing between two cylinders, $s / d=(b-a) / d$, for a motion trapped mode against $a / d$ with curves corresponding to (a).

reminiscent of curves of transmission coefficient for closely-spaced pairs of surface-piercing vertical barriers (see Porter 1995 or Evans \& Porter 1997, for example).

In figure $3(\mathrm{~b})$, we present a set of curves which have used the wide-spacing approximation, (2.3) to plot values of $s / d \equiv(b-a) / d$ against $a / d$ corresponding to those curves in figure 3(a). The ordinate $s / d$ represents half the (non-dimensional) predicted spacing between the interior vertical sides of a pair of rectangular motion trapping cylinders. Taking figures 3(a) and (b) together, allows one to read off, for any choice of $a / d$, the values of $k d$ and $s / d$ (or $b / d$ ) at which motion trapped modes are predicted to occur, on the basis of the wide-spacing approximation. Alternatively, we could have plotted $s / d$ against $k d$ instead of $a / d$ in figure $3(\mathrm{~b})$. When this is done, the data almost exactly collapses onto a single line, namely $k s=\pi$, making it impossible to distinguish the different data sets. This is not unexpected, since $k s=\pi$ corresponds to a single wavelength occupying the space between two vertical-sided rectangular cylinders and the resulting wave motion is approximately that of the fundamental symmetric sloshing mode between two vertical walls. Note that it is possible to use the wide-spacing approximation to predict lower values of the spacing than those shown in figure $3(\mathrm{~b})$, but as these clearly violate the widespacing assumption (which formally requires $k s \gg 1$ ) they are dismissed. We can also add other branches to figure 3(b) which represent larger spacings than those shown and correspond to a larger number of oscillations between the cylinders.

It is a simple matter to use the results obtained above for two-dimensional wave in- 
teractions with a cylinder of constant cross-section to predict the existence of a threedimensional axisymmetric motion trapped structure formed by the freely-heaving motion of a thick-walled floating cylindrical shell. There are again strong arguments for the existence of motion trapped modes here since it has been shown (Shipway \& Evans 2003) that there exist fixed body trapped modes in the vicinity of a pair of thin concentric shells. Just as for the closely-spaced fixed vertical barriers in two dimensions it is possible to use matched asymptotic expansions coupled with a wide-spacing approximation appropriate for axisymmetric problems to construct a trapped mode in the vicinity of closely-spaced concentric cylinders. But here too, the flow between the cylinders is slug-like and so once again, an example of fixed-body trapped modes in the vicinity of a pair of closely-spaced concentric cylinders can equally be regarded as a motion trapped mode interior to the heaving of a 'thin' thick-walled circular cylinder. An argument in favour can also be made using the fact that we have found that two-dimensional rectangular cylinders in heave are capable of reflecting an incident wave of a particular frequency. For thick cylinders of large radius $b$ a wide-spacing argument can be made for this axisymmetric problem in which the approximate form for the motion trapping frequency is given by a modified version of (2.3) in which an extra factor of $\pi / 4$ is added to the right-hand side. See for example, Shipway \& Evans (2003). Thus, the predicted relationship between $k d$ and $a / d$ for axisymmetric motion trapping structures of draft $d$ and inner to outer radius distance of $2 a$ remain as in figure $3(\mathrm{a})$. However, the predicted dependence of the inner radius of the circular shell, $r_{1}$, on $a / d$ can be inferred from figure $3(\mathrm{~b})$ via the relation $r_{1} / d=s / d+\pi /(4 k d)$.

\section{Exact methods for two dimensions}

The wide-spacing approximation provides strong evidence for the existence of a motion trapping structure in the form of a freely-floating catamaran formed by a pair of identical vertical rectangular cylinders whose centres are separated by a distance $2 b$. From symmetry it is clearly sufficient to consider a single freely heaving rectangular cylinder next to a vertical wall on which the velocity potential satisfies a Neumann condition, a process which gives rise to the approximate formula for the half-spacing $b$ in (2.3). To confirm the existence of motion trapped modes, an exact linear theory is required for this configuration from which the added mass and radiation damping coefficients can be determined and the conditions (2.1) and (2.2) checked. An obvious difficulty is 

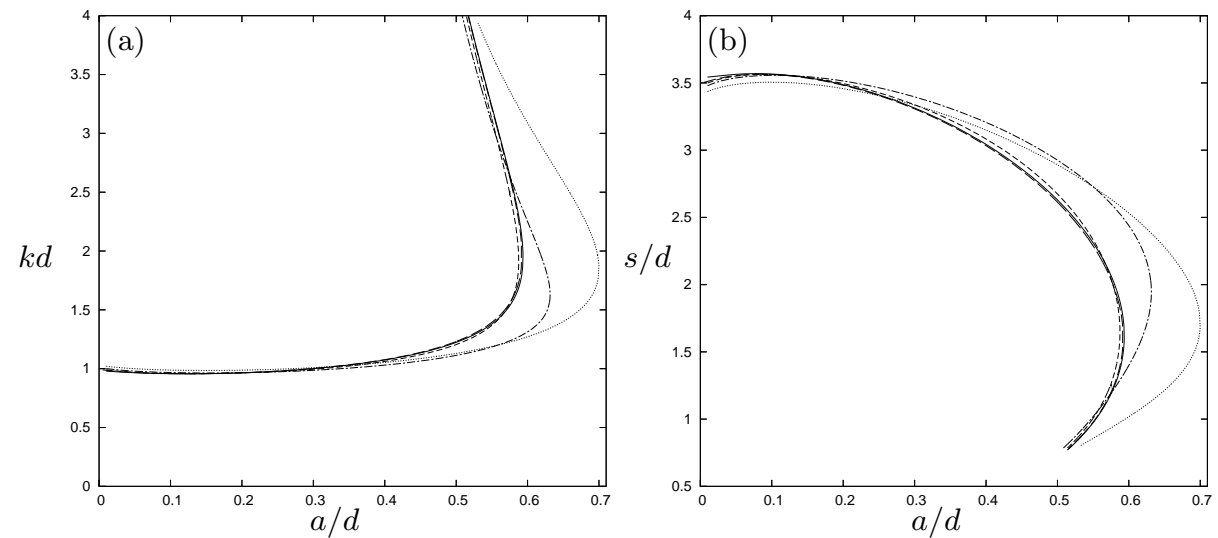

FiguRE 4. Exact parameters for generating motion trapped modes. In (a), the variation of $k d$ with $a / d$ with depth ratios $d / h=0.1$ (solid), 0.2 (long dash), 0.3 (short dash) and 0.4 (chained), 0.5 (dotted). In (b), the variation of half-spacing between two cylinders, $s / d=(b-a) / d$ with curves corresponding to (a).

that whereas condition (2.1) will be easily seen to be numerically satisfied when the left-hand-side changes sign, the same is not true of condition (2.2). Thus, the process of deciding numerically that the non-negative radiation damping actually takes the value zero is not an easy one. This difficulty can be overcome in the following manner. At those frequencies, if any, for which $b(\omega)=0$, there are no waves radiating to infinity and the velocity potential can, without loss of generality, be taken to be real. This means that in the eigenfunction expansion in the outer region extending to infinity, a real-valued term $a_{0} \sin k x$ or $a_{0} \cos k x, a_{0} \in \mathbb{R}$ can be substituted for the usual complex exponential term describing an outgoing wave, namely $a_{0} \mathrm{e}^{\mathrm{i} k x}, a_{0} \in \mathbb{C}$, where $a_{0}$ is the amplitude of the far field waves. Then the condition (2.2) gets replaced by the condition $a_{0}=0$ whilst (2.1) remains the same. The changes to the formulation, under such a revised scheme are minor only and outlined at the end of Appendix B. It is indeed confirmed numerically that at exactly those values at which $\nu=0$, the curves of $a_{0}$ against a frequency parameter do pass through zero from positive to negative values. Moreover, the existence of a crossing of the zero axis is robust to changes in numerical parameters which affect the accuracy of the results. This fact provides strong numerical evidence that the radiation damping does indeed vanish. A similar procedure was used in a recent related problem by the authors (Evans \& Porter, 2007).

In figures 3(a) and (b) the predicted relationships between the non-dimensional parameters $k d, s / d$ and $a / d$ for generating motion trapped modes were sketched as curves for 
different depth ratios $d / h$, the results being based on the wide-spacing approximation. In contrast, figures 4(a) and (b) now provide the exact values of $k d, s / d$ and $a / d$ for generating motion trapped modes, for a different (but overlapping) set of depth ratios of $d / h=0.1,0.2,0.3,0.4$ and 0.5 . Comparing the results of the two figures shows that there is only good agreement for $a / d$ smaller than about 0.4 . Whilst the curves for $d / h=0.1$ are qualitatively the same, the behaviour of the two sets of curves in figures 3 and 4 with increasing $d / h$ is altogether different, the exact results suggesting a maximum value of $a / d$ for motion trapped modes to exist for depths greater than the threshold value of $d / h=0.226$ given by the wide-spacing method. It is rare to observe the wide-spacing approximation perform so badly.

An explanation of the poor agreement between wide-spacing and exact results can be made with reference to the behaviour of the added mass and damping coefficients for two cylinders in heave at parameter values around the exact values. Thus, in figure 5 we fix (as a particular example) $d / h=0.2, a / d=0.5, b / d=3.11348$ and vary $k d$. The precise value of $b / d$ is chosen, because it implies (from our computations) that a motion trapped mode exists at $k d=1.23111$. It can be observed from figure $5(\mathrm{a})$, and in the magnified figure $5(\mathrm{~b})$, that there is a sharp and dramatic variation in both $\mu$ and $\nu$ (determined now from equation (B.7)) around the value of $k d=1.221$. The motion trapped mode is determined numerically by simultaneously requiring both the non-dimensional versions of $(2.1)$ and $(2.2)$, namely $f_{1}(k d) \equiv(1+\mu)-(K d)^{-1}$ and $\nu$, to be zero. As previously described, the latter condition can be traded in for $a_{0}=0$, provided the appropriate adjustments are made to the formulation. Thus, a motion trapped mode corresponds to the curves of $f_{1}$ and $a_{0}$, regarded as functions of $k d$, crossing the $k d$-axis at the same point, as shown in figure 5(b). The difficulty in getting both curves to cross at the same point is that the zero of $\nu$ is associated with the large variation in both $\nu$ and $\mu$ that occurs at a nearby value of $k d$ and is attributed to the existence of a pole of the function $\mu+\mathrm{i} \nu$ in the complex $k d$-plane close to the real line. Thus, it can be difficult to generate enough independent variation between the values of $\mu$ and $\nu$ close to the point at which $\nu=0$ in order to satisfy both conditions simultaneously.

Figures $6(\mathrm{a}, \mathrm{b})$ show the corresponding results for the thick axisymmetric cylinder derived from the theory described in Appendix $\mathrm{C}$ where the same device was used to track the trapped mode frequencies as described above in two dimensions. It can be seen that the figures are qualitatively similar to the two-dimensional case but the trapped modes 

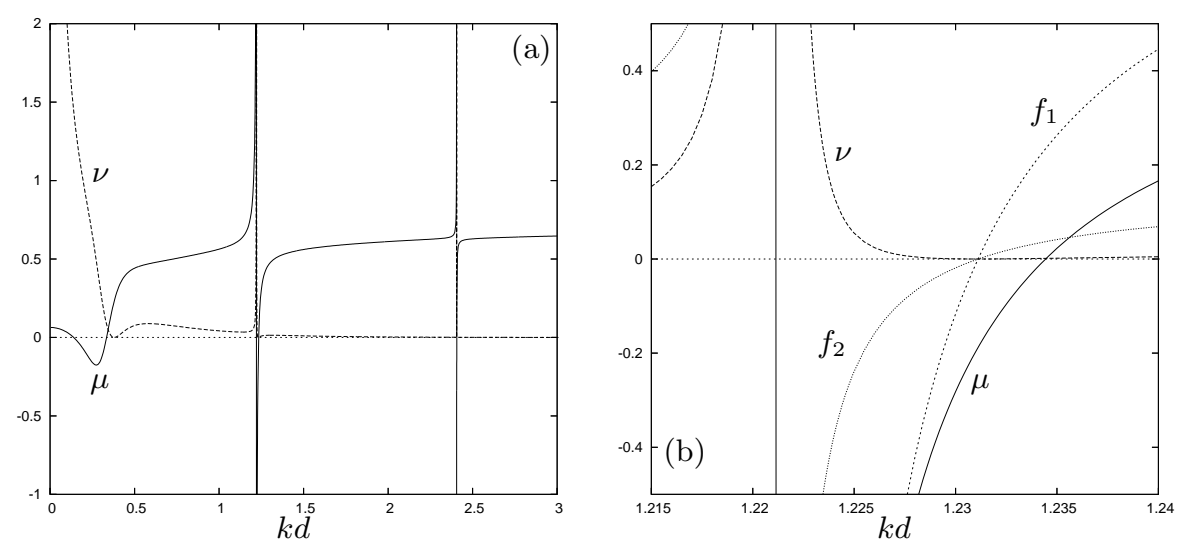

FiguRE 5. In (a), the variation of $\mu$ and $\nu$ with $k d$ for a motion trapped mode configuration of $d / h=0.2, a / d=0.5, b / d=3.11348$. In (b), a blow-up around the exact trapping wavenumber of $k d=1.23111$ with curves of $\mu$ and $\nu$ joined by curves of $f_{1}(k d) \equiv(1+\mu)-(K d)^{-1}$ and $f_{2}(k d) \equiv 10 a_{0}$.
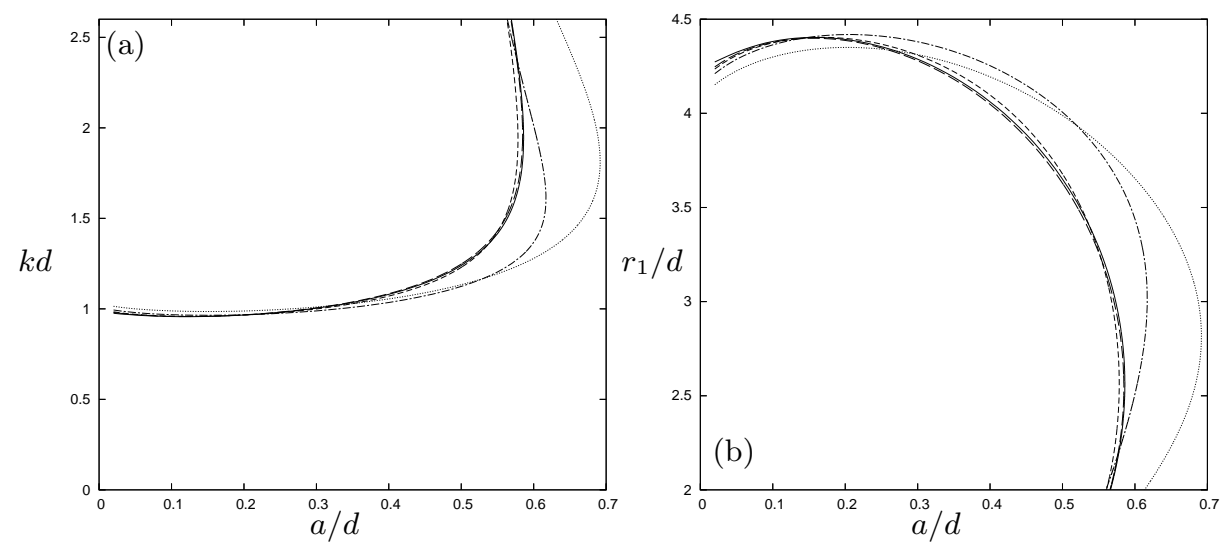

FIGURE 6. Exact parameters for generating motion trapped modes for axisymmetric circular shells of inner radius $r_{1}$, outer radius $r_{2}=r_{1}+2 a$ and draft $d$. In (a), the variation of $k d$ with $a / d$ with depth ratios $d / h=0.1$ (solid), 0.2 (long dash), 0.3 (short dash) and 0.4 (chained), 0.5 (dotted). In (b), the variation of inner cylinder radius, $r_{1} / d=(b-a) / d$ with curves corresponding to $(\mathrm{a})$.

arise for larger values of inner shell radius, $r_{1}$ than for half-spacing $s$ in that case. Some numerical inaccuracies associated with the computation of the axisymmetric problem meant that we only displayed a smaller range of results in figure 6 than in figure 5 .

\section{Conclusion}

It has been shown that one way of predicting pairs of motion trapping structures is to first find a single freely floating body which totally reflects an incident wave in its 
free motion. It was shown that it is necessary to have enough variable parameters in the problem to enable the zero transmission condition to be satisfied. Thus for a halfimmersed freely floating semicircular cylinder in heave this was not possible, but the extra geometric parameter of draft enabled zeroes of transmission to be found for a freely floating heaving vertical rectangular cylinder. Then wide-spacing arguments can be used to obtain an approximation for the spacing required for a motion trapping structure in motion at the zero-transmission frequency comprised of a pair of such cylinders. Exact multiple-interaction theory was used to confirm their existence and it was shown that the wide-spacing approximation does not always give reliable predictions for the values of frequency and spacing for motion trapped modes. A formulation was used to avoid difficulties in finding the zeroes of the non-negative radiation damping, replacing it by a separate condition which eliminates wave radiation to infinity and is both easily determined numerically and robust to inaccuracies in the numerical scheme. The ideas were extended to the case of a thick axisymmetric circular cylinder and results obtained from both an approximate and full linear theory obtained.

\section{Appendix A: Scattering and radiation by a single two-dimensional cylinder of rectangular cross-section}

First, we consider the numerical method used to compute the various quantities associated with a single fixed surface-piercing cylinder of rectangular cross-section of width $2 a$ and draft $d$ in fluid of constant depth $h$.

We are interested in (i) the reflection and transmission coefficients due to a wave incident on the fixed cylinder and (ii) the added-mass and radiation damping for the forced heave motion of the cylinder.

The method of solution is based upon using eigenfunction expansions in the rectangular subdomains of the fluid and matching across the interfaces (being those vertical lines extended from the sides of the cylinder down through the fluid). Using methods described in, for example, Porter (1995), integral equations are formulated for the horizontal component of velocity across the interfaces, which are subsequently approximated by a variational technique (equivalent to Galerkin's method) in which the anticipated singularities are incorporated into the approximation to the unknown velocities. This results in a linear system of equations, truncated to a finite size, $P$, whose chosen value determines the level of accuracy of the solutions. 
We suppress all detail of the formulation here, and simply provide the details sufficient to define the resulting numerical system.

First we need to make some definitions. Thus we have

$$
K=k \tanh k h=-k_{n} \tan k_{n} h, \quad \text { for } n=1,2, \ldots
$$

where $K=\omega^{2} / g, k$ is the single positive real root of the above and $k_{n}$ are an increasing sequence of positive real roots. Associated with these are

$$
N_{n}=\frac{1}{2}\left(1+\frac{\sin 2 k_{n} h}{2 k_{n} h}\right)
$$

which can be used for $n=0,1, \ldots$ with $k_{0}=-\mathrm{i} k$. Also,

$$
\mu_{n}=n \pi /(h-d), \quad n=0,1,2, \ldots
$$

We are required to determine the coefficients $\alpha_{p}^{(i)}, p=0, \ldots, P$ and $i=1,2,3$ from the system of equations

$$
\sum_{p=0}^{P} \alpha_{p}^{(i)} K_{p q}=F_{0 q}^{(i)}, \quad q=0, \ldots, P
$$

where

$$
K_{p q}=\sum_{n=1}^{\infty}\left[\frac{F_{n p}^{(1)} F_{n q}^{(1)}}{k_{n} h}+\frac{F_{n p}^{(2)} F_{n q}^{(2)}}{\mu_{n}(h-d) \tanh \mu_{n} a}\right]
$$

and

with

$$
F_{n p}^{(1)}=N_{n}^{-1 / 2} \frac{J_{2 p+1 / 6}\left(k_{n}(h-d)\right)}{\left[k_{n}(h-d)\right]^{1 / 6}}, \quad \text { for } n \geq 1
$$

$$
F_{0 p}^{(1)}=(-1)^{p} N_{0}^{-1 / 2} \frac{I_{2 p+1 / 6}(k(h-d))}{[k(h-d)]^{1 / 6}}
$$

where $J_{n}(\cdot), I_{n}(\cdot)$ are the Bessel function and modified Bessel function of the first kind. Also

$$
F_{n p}^{(2)}=\sqrt{2} \frac{J_{2 p+1 / 6}(n \pi)}{(n \pi)^{1 / 6}}, \quad \text { for } n \geq 1 \text { with } \quad F_{0 p}^{(2)}=\frac{2^{-1 / 6}}{\Gamma\left(\frac{7}{6}\right)} \delta_{0 p}
$$

where $\Gamma(\cdot)$ is the Gamma function. Also,

$$
F_{00}^{(3)}=\frac{9}{7} \frac{2^{-1 / 6}}{\Gamma\left(\frac{1}{6}\right)}, \quad F_{01}^{(3)}=-\frac{54}{91} \frac{2^{-1 / 6}}{\Gamma\left(\frac{1}{6}\right)}, \quad F_{0 p}^{(3)}=0, \quad \text { for } p \geq 2 .
$$

Using the values of $\alpha_{p}^{(i)}$ determined from (A.2) above we now define

$$
S_{i j}=\sum_{p=0}^{P} \alpha_{p}^{(i)} F_{0 p}^{(j)}, \quad i, j=1,2,3
$$

and it can be shown that $S_{i j}=S_{j i}$, a consequence of the underlying self-adjoint structure of the formulation. 
Then the reflection and transmission coefficients are defined by

$$
R=\frac{1}{2}\left(R^{s}+R^{a}\right), \quad T=\frac{1}{2}\left(R^{s}-R^{a}\right)
$$

where the two equations

$$
-\mathrm{i} k h\left(\mathrm{e}^{-\mathrm{i} k a}-R^{s} \mathrm{e}^{\mathrm{i} k a}\right)=\left(\mathrm{e}^{-\mathrm{i} k a}+R^{s} \mathrm{e}^{\mathrm{i} k a}\right) S_{11}-b_{0}^{s} S_{21}
$$

and

$$
0=\left(\mathrm{e}^{-\mathrm{i} k a}+R^{s} \mathrm{e}^{\mathrm{i} k a}\right) S_{12}-b_{0}^{s} S_{22},
$$

combine to allow $R^{s}$ and $b_{0}^{s}$ (a coefficient of no particular relevance) to be determined. To determine $R^{a}$, replaced $R^{s}$ and $b_{0}^{s}$ in (A.7) and (A.8) by $R^{a}$ and $b_{0}^{a}$ and solve a corresponding problem but with tanh $\mu_{n} a$ in (A.3) replaced by $\operatorname{coth} \mu_{n} a$ in the definition of $K_{p q}$.

The non-dimensional heave added mass, $\mu=a(\omega) / M$, and radiation damping, $\nu=$ $b(\omega) /(M \omega)$ are defined as the real and imaginary parts of

$$
\mu+\mathrm{i} \nu=\left(\frac{a}{d}\right)\left[\frac{1}{3} l^{-1}-b_{0}^{h}+l\left(\frac{2}{3}-A^{h} S_{13}+b_{0}^{h} S_{23}-l S_{33}\right)\right]
$$

where $l=(h-d) / a$ and

$$
\mathrm{i} k h A_{h}=A_{h} S_{11}-b_{0}^{h} S_{21}+l S_{31}
$$

combines with

$$
1=A_{h} S_{12}-b_{0}^{h} S_{22}+l S_{32}
$$

to determine $A_{h}$ and $b_{0}^{h}$. Here, $A_{h}$ corresponds to the far-field radiated wave amplitude in forced heaving of unit amplitude. The Haskind relation, in this context, translates to the identity $\nu=(a / d) k h\left|A_{h}\right|^{2}$.

\section{Appendix B: A pair of heaving two-dimensional cylinders of rectangular cross-section}

The method of solving for the heave added mass and radiation damping due to a rectangular cylinder of width $2 a$ and draft $d$, with centreline a distance $b$ from a vertical wall in fluid of finite depth $h$ is again based on eigenfunction expansion methods, similar to those used in Appendix A. Here, the situation is more complicated, in that expansions are required in three subdomains and matching occurs over two interfaces aligned with the vertical sections of the rectangular cylinder to yield a pair of coupled integral equations, 
which are solved via Galerkin's method. Again, we omit the algebraic details and provide only those details needed to compute the required added mass and radiation damping.

In matrix form, we are required to solve a system of linear equations for ten sets of coefficients, $\alpha_{p}^{(i)}, i=1, \ldots, 10$ which are expressed as the solution to

$$
\left(\begin{array}{cc}
K_{q p}^{(11)} & K_{q p}^{(12)} \\
K_{q p}^{(21)} & K_{q p}^{(22)}
\end{array}\right)\left(\begin{array}{ccccc}
\alpha_{p}^{(1)} & \alpha_{p}^{(3)} & \alpha_{p}^{(5)} & \alpha_{p}^{(7)} & \alpha_{p}^{(9)} \\
\alpha_{p}^{(2)} & \alpha_{p}^{(4)} & \alpha_{p}^{(6)} & \alpha_{p}^{(8)} & \alpha_{p}^{(10)}
\end{array}\right)=\left(\begin{array}{ccccc}
F_{0 q}^{(1)} & 0 & F_{0 q}^{(2)} & 0 & -F_{0 q}^{(3)} \\
0 & F_{0 q}^{(1)} & 0 & F_{0 q}^{(2)} & F_{0 q}^{(3)}
\end{array}\right)
$$

(summation is implied by repeated suffices). In the above,

$$
\begin{gathered}
K_{q p}^{(11)}=\sum_{n=1}^{\infty}\left\{\frac{\operatorname{coth} k_{n}(b-a)}{k_{n} h} F_{n p}^{(1)} F_{n q}^{(1)}+\frac{\operatorname{coth}\left(2 \mu_{n} a\right)}{n \pi} F_{n p}^{(2)} F_{n q}^{(2)}\right\} \\
K_{q p}^{(12)}=K_{q p}^{(21)}=-\sum_{n=1}^{\infty} \frac{\operatorname{cosech}\left(2 \mu_{n} a\right)}{n \pi} F_{n p}^{(2)} F_{n q}^{(2)}
\end{gathered}
$$

and

$$
K_{q p}^{(22)}=\sum_{n=1}^{\infty}\left\{\frac{1}{k_{n} h} F_{n p}^{(1)} F_{n q}^{(1)}+\frac{\operatorname{coth}\left(2 \mu_{n} a\right)}{n \pi} F_{n p}^{(2)} F_{n q}^{(2)}\right\}
$$

The factors $F_{n p}^{(i)}$ are the same as defined previously in (A.4)-(A.7). Once the coefficients $\alpha_{p}^{(i)}$ have been determined from (B.1), they are used to define the pair of $2 \times 2$ matrices $\mathrm{S}_{i 1}=\left(\begin{array}{cc}\alpha_{p}^{(1)} F_{0 p}^{(i 1)} & \alpha_{p}^{(3)} F_{0 p}^{(i 1)} \\ \alpha_{p}^{(2)} F_{0 p}^{(i 2)} & \alpha_{p}^{(4)} F_{0 p}^{(i 2)}\end{array}\right), \quad \mathrm{S}_{i 2}=\left(\begin{array}{cc}\alpha_{p}^{(5)} F_{0 p}^{(i 1)} & \alpha_{p}^{(7)} F_{0 p}^{(i 1)} \\ \alpha_{p}^{(6)} F_{0 p}^{(i 2)} & \alpha_{p}^{(8)} F_{0 p}^{(i 2)}\end{array}\right), \quad$ for $i=1,2$

(summation is implied by repeated suffices), with two-vectors

$$
\begin{gathered}
\mathbf{S}_{31}=\left(\begin{array}{c}
\alpha_{p}^{(2)} F_{0 p}^{(31)}-\alpha_{p}^{(1)} F_{0 p}^{(32)} \\
\alpha_{p}^{(4)} F_{0 p}^{(31)}-\alpha_{p}^{(3)} F_{0 p}^{(32)}
\end{array}\right), \quad \mathbf{S}_{32}=\left(\begin{array}{c}
\alpha_{p}^{(4)} F_{0 p}^{(31)}-\alpha_{p}^{(5)} F_{0 p}^{(32)} \\
\alpha_{p}^{(8)} F_{0 p}^{(31)}-\alpha_{p}^{(7)} F_{0 p}^{(32)}
\end{array}\right), \\
\mathbf{S}_{i 3}=\left(\begin{array}{c}
\alpha_{p}^{(9)} F_{0 p}^{(i 1)} \\
\alpha_{p}^{(10)} F_{0 p}^{(i 2)}
\end{array}\right), \quad i=1,2,
\end{gathered}
$$

and the coefficient $S_{33}=\alpha_{p}^{(10)} F_{0 p}^{(32)}-\alpha_{p}^{(9)} F_{0 p}^{(31)}$.

Next we define the pair of two-vectors

$$
\mathbf{A}_{1}=\left(\begin{array}{c}
-b_{0} \cos k(b-a) \\
a_{0}
\end{array}\right), \quad \mathbf{A}_{2}=\left(\begin{array}{c}
c_{0} \\
-c_{0}-2 d_{0}-2 / l
\end{array}\right)
$$

in terms of four coefficients $a_{0}, b_{0}, c_{0}$ and $d_{0}$ which are determined from the four linear equations

$$
\left(\begin{array}{c}
-k h b_{0} \sin k(b-a) \\
\mathrm{i} k h a_{0}
\end{array}\right)=\mathrm{S}_{11} \mathbf{A}_{1}+\mathrm{S}_{12} \mathbf{A}_{2}+l \mathbf{S}_{13}
$$


where $l=(h-d) / a$, combined with

$$
\left(\begin{array}{c}
d_{0} l \\
2+d_{0} l
\end{array}\right)=\mathrm{S}_{21} \mathbf{A}_{1}+\mathrm{S}_{22} \mathbf{A}_{2}+l \mathbf{S}_{23}
$$

The non-dimensional heave added-mass, $\mu$, and radiation damping, $\nu$, are then determined as the real and imaginary parts of

$$
\mu+\mathrm{i} \nu=-\left(\frac{a}{d}\right)\left[\frac{4}{3}\left(l^{-1}-l\right)+2 c_{0}+2 d_{0}+l\left\{\mathbf{S}_{31}^{\mathrm{T}} \mathbf{A}_{1}+\mathbf{S}_{32}^{\mathrm{T}} \mathbf{A}_{2}+l S_{33}\right\}\right]
$$

where the superscript $\mathrm{T}$ denotes the transpose.

For the purposes of locating zeros of damping, a numerically robust approach, outlined in section 4 , replaces the condition $\nu=0$ with $a_{0}=0$ where $a_{0}$ is now real and can take both positive and negative values ( $a_{0}$ is complex-valued in the system above).

The changes that the revised formulation enforces on the system above are trivial and involve replacing (B.5) by

$$
\mathbf{A}_{1}=\left(\begin{array}{c}
-b_{0} \cos k(b-a) \\
0
\end{array}\right)
$$

and the left-hand side of (B.6) with

$$
\left(\begin{array}{c}
-k h b_{0} \sin k(b-a) \\
k h a_{0}
\end{array}\right)
$$

Under this revised system, the resulting set of real coefficients $\left\{a_{0}, b_{0}, c_{0}, d_{0}\right\}$ only have a meaning when $a_{0}=0$ - i.e. can only be used to calculate $\mu$ when $a_{0}=0$.

\section{Appendix C: A heaving axisymmetric cylindrical shell of of rectangular cross-section}

We consider an axisymmetrical shell of draft $d$, inner radius $r_{1}=b-a$ and outer radius $r_{2}=b+a$ making heave oscillations in water of depth $h$. Our aim is to determine the heave added-mass and radiation damping. Again, the methods are based on eigenfunction expansions, now in cylindrical domains.

The structure of the equations to solve are the same as in (B.1) in Appendix B, but with revised definitions of the matrix coefficients:

$$
K_{q p}^{(11)}=\frac{r_{2}}{(h-d)} \sum_{n=1}^{\infty}\left\{\frac{I_{0}\left(k_{n} r_{1}\right)}{k_{n} h I_{1}\left(k_{n} r_{1}\right)} F_{n p}^{(11)} F_{n q}^{(11)}+\frac{\gamma_{n}\left(r_{1}, r_{2}\right)}{(n \pi) \Delta_{n}} F_{n p}^{(21)} F_{n q}^{(21)}\right\}
$$


and

$$
K_{q p}^{(12)}=-\sum_{n=1}^{\infty} \frac{F_{n p}^{(22)} F_{n q}^{(21)}}{(n \pi)^{2} \Delta_{n}}, \quad K_{q p}^{(21)}=-\sum_{n=1}^{\infty} \frac{F_{n p}^{(21)} F_{n q}^{(22)}}{(n \pi)^{2} \Delta_{n}}
$$

$$
K_{q p}^{(22)}=\frac{r_{1}}{(h-d)} \sum_{n=1}^{\infty}\left\{\frac{\gamma_{n}\left(r_{2}, r_{1}\right)}{(n \pi) \Delta_{n}} F_{n p}^{(22)} F_{n q}^{(22)}+\frac{K_{0}\left(k_{n} r_{2}\right)}{k_{n} h K_{1}\left(k_{n} r_{2}\right)} F_{n p}^{(12)} F_{n q}^{(12)}\right\}
$$

where $I_{n}(\cdot)$ and $K_{n}(\cdot)$ are modified Bessel functions whilst

$$
\gamma_{n}\left(r_{1}, r_{2}\right)=K_{0}\left(\mu_{n} r_{1}\right) I_{1}\left(\mu_{n} r_{2}\right)+K_{1}\left(\mu_{n} r_{2}\right) I_{0}\left(\mu_{n} r_{1}\right)
$$

and

$$
\Delta_{n}\left(r_{1}, r_{2}\right)=-I_{1}\left(\mu_{n} r_{1}\right) K_{1}\left(\mu_{n} r_{2}\right)+I_{1}\left(\mu_{n} r_{2}\right) K_{1}\left(\mu_{n} r_{1}\right)
$$

Definitions of $k_{n}, \mu_{n}$ and $F_{n p}^{(i)}, i=1,2,3$ can be found in Appendix A. Once the ten sets of coefficients $\alpha_{p}^{(i)}, i=1, \ldots, 10$ have been determined from (B.1) with the replacement for the matrix entries outlined above, then ' $\mathrm{S}$ ' matrices, vectors and coefficients are defined by the same set of equations (B.2)-(B.4) as in Appendix B.

Now define

$$
\mathbf{A}_{1}=\left(\begin{array}{c}
-b_{0} J_{0}\left(k r_{1}\right) \\
a_{0} H_{0}\left(k r_{2}\right)
\end{array}\right), \quad \text { and } \quad \mathbf{A}_{2}=\left(\begin{array}{c}
c_{0} \\
-c_{0}-d_{0} \log \left(r_{2} / r_{1}\right)-\lambda
\end{array}\right) .
$$

where

$$
\lambda=\frac{a b}{(h-d)^{2}}-\frac{r_{1}^{2}}{2(h-d)^{2}} \log \left(\frac{r_{2}}{r_{1}}\right) .
$$

in which the set of four coefficients $\left\{a_{0}, b_{0}, c_{0}, d_{0}\right\}$ are determined from the equations

$$
\left(\begin{array}{c}
-\left[k h(h-d) J_{1}\left(k r_{1}\right) / r_{2}\right] b_{0} \\
-\left[k h(h-d) H_{1}\left(k r_{2}\right) / r_{1}\right] a_{0}
\end{array}\right)=\mathrm{S}_{11} \mathbf{A}_{1}+\mathrm{S}_{12} \mathbf{A}_{2}+\mathbf{S}_{13}
$$

combined with

$$
\left(\begin{array}{c}
(h-d)^{2} d_{0} /\left(r_{1} r_{2}\right) \\
2 a b /\left(r_{1} r_{2}\right)+(h-d)^{2} d_{0} /\left(r_{1} r_{2}\right)
\end{array}\right)=\mathrm{S}_{21} \mathbf{A}_{1}+\mathrm{S}_{22} \mathbf{A}_{2}+\mathbf{S}_{23}
$$

The non-dimensional heave added-mass, $\mu$ and radiation damping $\nu$ are given by

$$
\mu+\mathrm{i} \nu=-\frac{1}{2 a b d}\left(C_{1}+C_{2}+C_{3}\right)
$$

where

$$
C_{1}=-a b(h-d)+\frac{a b\left(b^{2}+a^{2}\right)}{2(h-d)}-\frac{r_{1}^{2} r_{2}^{2} \log \left(r_{2} / r_{1}\right)}{4(h-d)}
$$

with

$$
C_{2}=2 a b(h-d)\left[c_{0}+d_{0} \log \left(r_{2} / r_{1}\right)+\lambda-\frac{1}{6}\right]
$$


and

$$
C_{3}=r_{1} r_{2}(h-d)\left\{\mathbf{S}_{31}^{\mathrm{T}} \mathbf{A}_{1}+\mathbf{S}_{32}^{\mathrm{T}} \mathbf{A}_{2}+S_{33}\right\}-(h-d)\left[2 a b+d_{0}(h-d)^{2}\right] \lambda .
$$

In order to numerically determine frequencies at which zeroes of damping occur, it is more convenient to revise the system presented above in a manner similar to that described at the end of Appendix B. Thus, (C.1) is replaced by

$$
\mathbf{A}_{1}=\left(\begin{array}{c}
-b_{0} J_{0}\left(k r_{1}\right) \\
a_{0} J_{0}\left(k r_{2}\right)
\end{array}\right)
$$

and the left-hand-side of (C.2) is replaced with

$$
\left(\begin{array}{c}
-\left[k h(h-d) J_{1}\left(k r_{1}\right) / r_{2}\right] b_{0} \\
-\left[k h(h-d) J_{1}\left(k r_{2}\right) / r_{1}\right] a_{0}
\end{array}\right) .
$$

Under this revised system, the coefficients $\left\{a_{0}, b_{0}, c_{0}, d_{0}\right\}$ are all real, and $\nu=0$ is equivalent to $a_{0}=0$ where $a_{0}$ can take both positive and negative values.

\section{REFERENCES}

Evans, D.V. \& Fernyhough, M., 1995, Edge waves along periodic coastlines. Part 2. J. Fluid. Mech. 297, 301-325.

Evans, D.V. \& Linton, C.M., 1989, Active devices for the reduction of wave intensity. Appl. Ocean Res., 11, 26-32.

Evans, D.V. \& Kuznetsov, N., 1997, Trapped modes. Gravity waves in water of finite depth. Southampton: Computational Mechanics. pp.127-168.

Evans, D.V. \& MorRis, C.A.N., 1972, Complementary approximations to the solution of a problem in water waves. J. Inst. Appl. Maths. Applics., 9, 1-10.

Evans, D.V. \& PorTer, R. 1997, Complementary methods for scattering by thin barriers In Mathematical Techniques for Water Waves. Ed. B.N. Mandal, Computational Mechanics Publications, Southampton.

Evans, D.V. \& Porter, R., 2007, Wave free motions of isolated bodies and the existence of motion trapped modes. J. Fluid. Mech. 584, 225-234

Martin, P.A. \& Dixon, A.G., 1983, The scattering of regular surface waves by a fixed, halfimmersed, circular cylinder. Appl. Ocean Res., 5(1), 13-23.

McIver, M., 1996, An example of non-uniqueness in the two-dimensional linear water wave problem. J. Fluid Mech., 315, 257-266.

McIver, P. \& McIver, M., 2006, Trapped modes in the water-wave problem for a freely floating structure. J. Fluid Mech., 558, 53-67.

McIver, P. \& McIver, M., 2007, Motion trapping structures in the three-dimensional waterwave problem. J. Eng. Math., 58, 67-75. 
Newman, J.N., 1974, Interaction of water waves with two closely-spaced barriers. J. Fluid Mech., 66, 97-106.

Porter, R., 1995, Complementary methods and bounds in linear water waves. Ph.D. thesis, University of Bristol.

Shipway, B.J. \& Evans, D.V., 2003, Wave trapping by axisymmetric concentric cylinders. J. Offshore Mech. Arctic Eng., 125(1), 59-64.

Yeung, R.W. \& SEAH, R.K.M., 2007, On Helmholtz and higher-order resonance of twin floating bodies. J. Eng. Math., 58, 251-265.

WANG, S. \& Wahab, R., 1971, Heaving oscillations of twin cylinders in a free surface. J. Ship Res., 15(1), 33-48. 